\title{
Sensitivity Analysis for Averaged Asset Price Dynamics with Gamma Processes
}

\author{
REIICHIRO KAWAI* AND ATSUSHI TAKEUCHI ${ }^{\dagger}$
}

\begin{abstract}
The main purpose of this paper is to derive unbiased Monte Carlo estimators of various sensitivity indices for an averaged asset price dynamics governed by the gamma Lévy process. The key idea is to apply a scaling property of the gamma process with respect to the Esscher density transform parameter. Our framework covers not only the continuous Asian option, but also European, discrete Asian, average strike Asian, weighted average, spread options, and geometric average Asian options. Numerical results are provided to illustrate the effectiveness of our formulas in Monte Carlo simulations, relative to finite difference approximation.

Keywords: Asian options, Esscher transform, gamma process, Greeks, Lévy process, Malliavin calculus.
\end{abstract}

2000 Mathematics Subject Classification: 60E07, 60G51, 91B70, 60H07.

\section{Introduction}

In mathematical finance, it has been widely known that the logarithmic derivatives of the density function of stochastic differential equations correspond essentially to the so-called Greeks, that is, sensitivity indices with respect to various model parameters of asset price dynamics. The pioneer work in this direction is of Fournié et al. [11], whose approach is based upon the integration-byparts formula developed in the Malliavin calculus on the Wiener space. They provide a systematic approach to the derivation of various Greeks formulas for a relatively general diffusion asset price

Published in Statistics and Probability Letters (2010) 80(1) 42-49.

*Email Eddress: reiichiro.kawai@gmail.com. Postal Address: Department of Mathematics, University of Leicester, Leicester LE1 7RH, UK.

Corresponding Author. Email Address: takeuchi@sci.osaka-cu.ac.jp. Postal Address: Department of Mathematics, Osaka City University, Sugimoto 3-3-138, Osaka 558-8585, Japan. 
dynamics. Since then, there has long been a natural and nontrivial question whether a similar Malliavin calculus approach is applicable in the case of jump processes.

The investigation of the Malliavin calculus for jump processes is initiated in Bismut [4] based upon the Girsanov density transform with a view towards the study of the existence and the smoothness of the density. Various types of Malliavin calculus and logarithmic derivatives have been studied on the Poisson space or on the Wiener-Poisson space. In particular, the existence of weights for the logarithmic derivatives dates back to the work of Bichteler, Gravereaux and Jacod [3], while the uniqueness and the closed forms of the weight have not been established in the general framework.

For practical considerations, however, it is most important to have closed, but not necessarily unique, weights on hand, in order to design an efficient Monte Carlo evaluation. In this direction, Greeks formulas are obtained in Davis and Johansson [9] and Cass and Friz [8] for jump diffusion processes. Their approach consists of conditioning on the jump component and then performing the Malliavin calculus techniques on the diffusion component. In other words, their models are required to be a superposition of independent jump and diffusion components, where the diffusion one must not be degenerate. On the other hand, El-Khatib and Privault [10] applied the Malliavin calculus focused on the Poisson arrival times due to Carlen and Pardoux [5], while Bally et al. [1] took a unified approach considering the derivatives with respect to both the Poisson arrival times and the amplitude of the jumps. Kawai and Kohatsu-Higa [13] derives formulas for Lévy process models of time-changed Brownian motion type, by employing the Gaussian Malliavin calculus conditionally on the time-changing process. Takeuchi [17] studied the same problem for the stochastic differential equations with jumps via a martingale approach. Kawai and Takeuchi [14] studied the computation of the Greeks of European payoffs for an asset price dynamics defined with gamma processes and with Brownian motions, possibly time-changed by one of the gamma processes. In Kawai and Takeuchi [14], various well known financial models, such as the BlackScholes model and the variance gamma model of Madan, Carr and Chang [15], are within its framework. The key idea there is to apply a scaling property of the gamma distribution with respect to the Esscher transform.

In this paper, we take an approach again based on the scaling property, not of the gamma distribution as in Kawai and Takeuchi [14], but of the gamma Lévy process. This, rather simple, extension widens the applicability to the averaged asset price dynamics defined with the gamma process. A typical example of interest is the (continuous) Asian option, which looks at the time average of underlying asset price dynamics, unlike only the terminal value in the European options. Meanwhile, many others, such as European, discrete Asian, average strike Asian, weighted average and spread options, can easily be accommodated in our framework. In addition, Asian options of geometric average type are also within our scope. This work can also be thought of as 
a considerable generalization of Kawai and Takeuchi [14], in the sense that some of the European Greeks formulas there can be recovered from our results. Finally, our approach taken in this paper can also be applied in various stochastic systems involving gamma processes.

The rest of the paper is organized as follows. Section 2 recalls generalities on the gamma process and introduces our asset price dynamics model with assumptions to be imposed on its characterizing parameters. In Section 3, we derive formulas of the Greeks for discontinuous payoff function of averaged asset price dynamics defined with the gamma process. The derivation of our formulas entails rather lengthy proofs of somewhat routine nature. To avoid overloading the paper, we omit nonessential details in some instances. We close this study with some numerical results to illustrate remarkable improvements in Monte Carlo simulations in terms of estimator variance relative to the finite difference estimation.

\section{Preliminaries}

Let us begin with general notations which will be used throughout the text. For each positive integer $k, \partial_{k}$ indicates the partial derivative with respect to $k$-th $\operatorname{argument}$. We fix $(\Omega, \mathscr{F}, \mathbb{P})$ as our underlying the probability space. We denote by $\mathbb{E}_{\mathbb{P}}$ the expectation under the probability measure $\mathbb{P}$. We denote by $\delta_{x}(d y)$ the Dirac delta measure on $\mathbb{R}$ with concentration at $x \in \mathbb{R}$, while $B_{\varepsilon}:=(-\varepsilon,+\varepsilon)$ is the open ball around the origin with radius $\varepsilon>0$. Let $\left\{Y_{t}: t \geq 0\right\}$ be a one-sided pure-jump Lévy process in $[0,+\infty)$ with the Lévy measure

$$
v(d z)=a \frac{e^{-b z}}{z} d z, \quad z \in(0,+\infty),
$$

where $a>0$ and $b>0$. The stochastic process $\left\{Y_{t}: t \geq 0\right\}$ is then called a gamma process with parameter $(a, b)$, whose marginal has the gamma distribution with characteristic function

$$
\mathbb{E}_{\mathbb{P}}\left[e^{i y Y_{t}}\right]=\exp \left[t \int_{(0,+\infty)}\left(e^{i y z}-1\right) v(d z)\right]=\left(1-\frac{i y}{b}\right)^{-a t}
$$

Moreover, the marginal density function at time $t$ is given in the form

$$
f_{t}^{\mathbb{P}}(y)=\frac{b^{a t}}{\Gamma(a t)} y^{a t-1} e^{-b y}, \quad y \in(0,+\infty),
$$

where $\Gamma(p)$ is the gamma function of order $p>0$.

Throughout the paper, we fix $T>0$. Define $\Lambda:=\left\{\lambda \in \mathbb{R}: \mathbb{E}_{\mathbb{P}}\left[e^{\lambda Y_{1}}\right]<+\infty\right\}=(-\infty, b)$. For $\lambda \in \Lambda$, define a new probability measure $\mathbb{Q}_{\lambda}$ by

$$
\left.\frac{d \mathbb{Q}_{\lambda}}{d \mathbb{P}}\right|_{\mathscr{F}_{T}}:=\frac{e^{\lambda Y_{T}}}{\mathbb{E}_{\mathbb{P}}\left[e^{\lambda Y_{T}}\right]}=\exp \left[\lambda Y_{T}-\varphi_{T}(\lambda)\right],
$$


where $\varphi_{T}(\lambda):=a T \ln (b /(b-\lambda))$ and where $\left(\mathscr{F}_{t}\right)_{t \in[0, T]}$ is the natural filtration generated by $\left\{Y_{t}\right.$ : $t \in[0, T]\}$. This measure change is the simplest form of the Girsanov transformation, often called the Esscher transform in mathematical finance and actuarial science. The following is the key tool for our discussions.

Lemma 2.1. The laws of the process $Y$ under $\mathbb{Q}_{\lambda}$ and of the process $Y^{(\lambda)}:=b Y /(b-\lambda)$ under $\mathbb{P}$ are identical.

Proof. It is well known that for Lévy processes, the identity of finite dimensional distributions implies the identity of random processes in law. Fix $0=t_{0} \leq t_{1} \leq \cdots \leq t_{n}=T$ and $\zeta_{1}, \cdots, \zeta_{n} \in \mathbb{R}$ arbitrarily. Due to the independence of the increments under the measure $\mathbb{P}$, we have

$$
\begin{aligned}
\mathbb{E}_{\mathbb{Q}_{\lambda}}\left[\exp \left(i \sum_{k=1}^{n} \zeta_{k} Y_{t_{k}}\right)\right] & =\mathbb{E}_{\mathbb{P}}\left[\exp \left(i \sum_{k=1}^{n} \zeta_{k} Y_{t_{k}}+\lambda Y_{T}\right)\right]\left(1-\frac{\lambda}{b}\right)^{a T} \\
& =\prod_{j=1}^{n}\left(1-\frac{i \sum_{k=j}^{n} \zeta_{k}+\lambda}{b}\right)^{-a\left(t_{j}-t_{j-1}\right)}\left(1-\frac{\lambda}{b}\right)^{a\left(t_{j}-t_{j-1}\right)} \\
& =\mathbb{E}_{\mathbb{P}}\left[\exp \left(i \sum_{k=1}^{n} \zeta_{k} \frac{b}{b-\lambda} Y_{t_{k}}\right)\right]
\end{aligned}
$$

which proves the claim.

Let us introduce our asset price dynamics model and with some examples of practical interest within our scope. Let $Z:=\left\{Z_{t}: t \in[0, T]\right\}$ be a nondecreasing stochastic process in $\mathbb{R}$ with $Z_{0}=0$, $\mathbb{P}$-a.s., independent of the process $Y:=\left\{Y_{t}: t \in[0, T]\right\}$. Our asset price dynamics model $\left\{S_{t}: t \in\right.$ $[0, T]\}$ is defined by

$$
S_{t}:=S_{0} \exp \left[\theta Y_{t}+\kappa Z_{t}+c(\theta, \kappa) t\right],
$$

where $S_{0} \in(0,+\infty), \theta \in(0,+\infty), \kappa \in \mathbb{R}$, and $c:(0,+\infty) \times \mathbb{R} \rightarrow \mathbb{R}$ with $\partial_{k} c(\theta, \kappa)$ being well defined for $k=1,2$. Let us impose some assumptions on the parameters $\theta$ and $b$ and on the process $Z$.

Assumption 2.2. We henceforth assume the following;

(i) $4 \theta<b$,

(ii) there exists a constant $p_{0}>2$ such that $\mathbb{E}_{\mathbb{P}}\left[\exp \left(p_{0}|\kappa| Z_{T}\right)\right]<+\infty$.

Remark 2.3. Let us propose some candidates for the process $Z$, which makes our asset price dynamics model of adequate practical interest. 
The first one is the variance gamma model of Madan, Carr and Chang [15] by setting $Z$ also to be a gamma process with a negative $\kappa$. The model has attracted the attention of finance practitioners, and have thus appeared often in the computational finance literature, for example, Carr and Madan [7] and Fu [12]. To be precise, set $\theta=1$ and $\kappa=-1$, and the gamma processes $Y$ and $Z$ should be characterized respectively with $(a, b)=\left(v^{-1},\left(\mu_{p} v\right)^{-1}\right)$ and $(a, b)=\left(v^{-1},\left(\mu_{n} v\right)^{-1}\right)$ for some $v>0$, where $\mu_{p}=\frac{1}{2} \sqrt{\theta^{2}+2 \sigma^{2} / v}+\theta / 2$, and $\mu_{n}=\frac{1}{2} \sqrt{\theta^{2}+2 \sigma^{2} / v}-\theta / 2$.

The inverse Gaussian process is another interesting candidate for $Z$. It is also a subordinator, induced by the inverse Gaussian distribution, whose marginal at time $t$ is identical in law to the first time that a Brownian motion with drift $v$ reaches the positive level $t$, that is, $\mathbb{E}_{\mathbb{P}}\left[e^{-y Z_{t}}\right]=\exp [-$ $\left.t\left(\sqrt{2 y+v^{2}}-v\right)\right]$. More details on the inverse Gaussian process may be found in BarndorffNielsen [2] and Carr et al. [6].

\section{Sensitivity formulas}

Let $\mu(d t)$ be a finite Borel measure over $[0, T]$. For simplicity in notation, we henceforth write

$$
\begin{aligned}
S_{t}^{(\lambda)} & :=S_{0} \exp \left[\theta Y_{t}^{(\lambda)}+\kappa Z_{t}+c(\theta, \kappa) t\right], \quad F_{j}^{(\lambda)}:=\frac{1}{T} \int_{0}^{T} S_{t}^{(\lambda)}\left(Y_{t}^{(\lambda)}\right)^{j} \mu(d t) \quad(j=0,1,2,3), \\
H_{j}^{(\lambda)} & :=\frac{1}{T} \int_{0}^{T} S_{t}^{(\lambda)}\left(Y_{t}^{(\lambda)}\right)^{j} Z_{t} \mu(d t), \quad I_{j}^{(\lambda)}:=\frac{1}{T} \int_{0}^{T} S_{t}^{(\lambda)}\left(Y_{t}^{(\lambda)}\right)^{j} t \mu(d t) \quad(j=0,1), \\
J^{(\lambda)} & :=\frac{1}{T} \int_{0}^{T} S_{t}^{(\lambda)} Z_{t} t \mu(d t), \quad K^{(\lambda)}:=\left(b Y_{T}^{(\lambda)}-a T+1\right) \frac{F_{0}^{(\lambda)}}{\theta F_{1}^{(\lambda)}}+\frac{F_{0}^{(\lambda)} F_{2}^{(\lambda)}}{\left(F_{1}^{(\lambda)}\right)^{2}}-1, \\
L^{(\lambda)} & :=\left(b Y_{T}^{(\lambda)}-a T+1\right) \frac{I_{0}^{(\lambda)}}{\theta F_{1}^{(\lambda)}}+\frac{I_{0}^{(\lambda)} F_{2}^{(\lambda)}}{\left(F_{1}^{(\lambda)}\right)^{2}}-\frac{I_{1}^{(\lambda)}}{F_{1}^{(\lambda)}}, \\
M^{(\lambda)} & :=\left(b Y_{T}^{(\lambda)}-a T+1\right) \frac{H_{0}^{(\lambda)}}{\theta F_{1}^{(\lambda)}}+\frac{H_{0}^{(\lambda)} F_{2}^{(\lambda)}}{\left(F_{1}^{(\lambda)}\right)^{2}}-\frac{H_{1}^{(\lambda)}}{F_{1}^{(\lambda)}}
\end{aligned}
$$

for $\lambda \in \Lambda$ and $t \in[0, T]$. For further simplicity, when $\lambda=0$, we suppress the superscript (0) of $Y_{t}^{(0)}, S_{t}^{(0)}, F_{j}^{(0)}, H_{j}^{(0)}, I_{j}^{(0)}, J^{(0)}, K^{(0)}, L^{(0)}$ and $M^{(0)}$. We begin with our main results.

Theorem 3.1. Let $\Phi: \mathbb{R}_{+} \rightarrow \mathbb{R}$ be a measurable function such that $\mathbb{E}_{\mathbb{P}}\left[\left|\Phi\left(F_{0}\right)\right|^{2}\right]$ is locally uniformly bounded in $S_{0}, \theta$ and $\kappa$. Then, we have

(i) [Sensitivity with respect to $S_{0}$ ]

$$
\frac{\partial}{\partial S_{0}} \mathbb{E}_{\mathbb{P}}\left[\Phi\left(F_{0}\right)\right]=\frac{1}{S_{0}} \mathbb{E}_{\mathbb{P}}\left[\Phi\left(F_{0}\right) K\right] .
$$


(ii) [Second derivative with respect to $S_{0}$ ]

$$
\begin{gathered}
\frac{\partial^{2}}{\partial S_{0}^{2}} \mathbb{E}_{\mathbb{P}}\left[\Phi\left(F_{0}\right)\right]=\frac{1}{S_{0}^{2}} \mathbb{E}_{\mathbb{P}}\left[\Phi ( F _ { 0 } ) \left(3\left(b Y_{T}-a T+1\right) K \frac{F_{0}}{\theta F_{1}}\right.\right. \\
\left.\left.-\left(2\left(b Y_{T}-a T+1\right)^{2}+a T-1\right)\left(\frac{F_{0}}{\theta F_{1}}\right)^{2}+1-\frac{F_{0}^{2} F_{3}}{F_{1}^{3}}+3 \frac{F_{0}^{2} F_{2}^{2}}{F_{1}^{4}}-3 \frac{F_{0} F_{2}}{F_{1}^{2}}\right)\right]-\frac{1}{S_{0}} \frac{\partial}{\partial S_{0}} \mathbb{E}_{\mathbb{P}}\left[\Phi\left(F_{0}\right)\right] .
\end{gathered}
$$

(iii) [Sensitivity with respect to $\theta$ ]

$$
\frac{\partial}{\partial \theta} \mathbb{E}_{\mathbb{P}}\left[\Phi\left(F_{0}\right)\right]=\mathbb{E}_{\mathbb{P}}\left[\Phi\left(F_{0}\right)\left(\frac{b Y_{T}-a T}{\theta}+\partial_{1} c(\theta, \kappa) L\right)\right] .
$$

(iv) [Sensitivity with respect to $\kappa$ ]

$$
\frac{\partial}{\partial \kappa} \mathbb{E}_{\mathbb{P}}\left[\Phi\left(F_{0}\right)\right]=\mathbb{E}_{\mathbb{P}}\left[\Phi\left(F_{0}\right)\left(M+\partial_{2} c(\theta, \kappa) L\right)\right]
$$

Remark 3.2. In the context of mathematical finance, the sensitivity (i) and (ii) are called Delta and Gamma, respectively. Both of the formulas (iii) and (iv) correspond best to the Vega, that is, the sensitivity with respect to the Black-Scholes volatility parameter.

Our framework covers several payoffs of practical interest. First, setting $\mu(d t)=T \delta_{T}(d t)$ reduces to the European framework. With this setting, our results indeed recover the European formulas derived in Kawai and Takeuchi [14]. As a matter of course, $\mu(d t)=d t$ corresponds to the Asian option (also called average price option), while in real financial trading, the continuous average is simply impossible to compute and is thus not very interesting. Meanwhile, we can deal with arithmetic average (discrete Asian) type, which is rather the genuine Asian from a practical point of view, by setting $\mu(d t)=T N^{-1} \sum_{k=1}^{N} \delta_{t_{k}}(d t)$, to be numerically tested later, or a little more generally, a weighted arithmetic average by $\mu(d t)=T\left(\sum_{k=1}^{N} w_{k}\right)^{-1} \sum_{k=1}^{N} w_{k} \delta_{t_{k}}(d t)$ for reals $\left\{w_{k}\right\}_{k \in \mathbb{N}}$. Options of average strike Asian type and of spread type, respectively with $\mu(d t)=$ $d t-T \delta_{T}(d t)$ and $\mu(d t)=T\left(-\delta_{T / 2}(d t)+\delta_{T}(d t)\right)$, are also within our framework.

The proof of Theorem 3.1 is divided into several preliminary results. The first one is concerned with the moments of $Y_{t}^{(\lambda)}$ and of $S_{t}^{(\lambda)}$.

Lemma 3.3. Fix $t \in[0, T]$ and $\lambda \in \Lambda$. It holds that for $p \in(-a t,+\infty)$,

$$
\mathbb{E}_{\mathbb{P}}\left[\left(Y_{t}^{(\lambda)}\right)^{p}\right]=\frac{\Gamma(a t+p)}{(b-\lambda)^{p} \Gamma(a t)}
$$

while for $p \in\left(-\infty, p_{0} \wedge(b-\lambda) / \theta\right)$,

$$
\mathbb{E}_{\mathbb{P}}\left[\left(S_{t}^{(\lambda)}\right)^{p}\right]=\left(1-\frac{p \theta}{b-\lambda}\right)^{-a t} S_{0}^{p} \exp (p c(\theta, \kappa) t) \mathbb{E}_{\mathbb{P}}\left[\exp \left(p \kappa Z_{t}\right)\right]
$$


Proof. The first assertion is straightforward by the density (2.1), while the second follows from the independence of $Y$ and $Z$, the exponential integrability of $Z$ in Assumption 2.2, and again the density (2.1).

We fix $\varepsilon \in(0, b / 2)$ in what follows. Note that $B_{\varepsilon} \subset \Lambda$, and $p_{0} \wedge(b-\lambda) / \theta>2$ under Assumption 2.2. The following lemma later plays an important role for the the interchange of the derivative (with respect to $\lambda$ ) and the expectation $\mathbb{E}_{\mathbb{P}}$.

Lemma 3.4. The random variables $S_{t}^{(\lambda)}$ and $Y_{t}^{(\lambda)}$ are in $\mathbb{L}^{p_{0} \wedge(b-\lambda) / \theta}(\Omega)$, uniformly in $t \in[0, T]$ and $\lambda \in B_{\varepsilon}$.

Proof. The assertion is the direct consequence of Assumption 2.2 and Lemma 3.3.

The following lemma is also an important tool.

Lemma 3.5. For $k=0,1,2$ and for $\lambda \in B_{\varepsilon}$, it holds that

$$
\frac{\partial}{\partial \lambda} \int_{0}^{T} S_{t}^{(\lambda)}\left(Y_{t}^{(\lambda)}\right)^{k} Z_{t} \mu(d t)=\int_{0}^{T} \frac{\partial}{\partial \lambda}\left(S_{t}^{(\lambda)}\left(Y_{t}^{(\lambda)}\right)^{k}\right) Z_{t} \mu(d t), \quad \mathbb{P} \text {-a.s. }
$$

Proof. Clearly, for each $t \in[0, T], M_{t}^{(\lambda)}:=S_{t}^{(\lambda)}\left(Y_{t}^{(\lambda)}\right)^{k} Z_{t}$ is continuous in $\lambda$, so is $(\partial / \partial \lambda) M_{t}^{(\lambda)}=$ $M_{t}^{(\lambda)}\left(\theta Y_{t}^{(\lambda)}+k\right) /(b-\lambda)$. Since $Y_{t}^{(\lambda)}$ is nondecreasing in $t$, we have for $h \in(0, b-\lambda)$,

$$
\begin{aligned}
& \int_{0}^{T} \int_{0}^{1}\left(\left.\frac{\partial}{\partial \xi} M_{t}^{(\xi)}\right|_{\xi=\lambda+\delta h}\right)^{2} d \delta \mu(d t) \\
& \leq\left. S_{0}^{2} \exp \left(2 \theta Y_{T}^{(\xi)}+2|\kappa| Z_{T}+2|c(\theta, \kappa)| T\right)\left(Y_{T}^{(\xi)}\right)^{2 k} Z_{T}^{2}\left(\frac{\theta Y_{T}^{(\xi)}+k}{b-\xi}\right)^{2} \mu([0, T])\right|_{\xi=\lambda+h} .
\end{aligned}
$$

By the Taylor theorem, it holds that

$$
\begin{aligned}
\frac{1}{h}\left(\int_{0}^{T} M_{t}^{(\lambda+h)} \mu(d t)-\int_{0}^{T} M_{t}^{(\lambda)} \mu(d t)\right)- & \int_{0}^{T} \frac{\partial}{\partial \lambda} M_{t}^{(\lambda)} \mu(d t) \\
& =\int_{0}^{T} \int_{0}^{1}\left(\left.\frac{\partial}{\partial \xi} M_{t}^{(\xi)}\right|_{\xi=\lambda+\delta h}-\frac{\partial}{\partial \lambda} M_{t}^{(\lambda)}\right) \mu(d t)
\end{aligned}
$$

which tends to 0 as $h \downarrow 0$. The proof is complete.

Let us investigate the integrability of the random variable $F_{1}^{(\lambda)}$ of negative order.

Lemma 3.6. For $p \in\left(1,\left(p_{0} \wedge a T\right) / 2\right)$, it holds that

$$
\sup _{\lambda \in B_{\varepsilon}} \mathbb{E}_{\mathbb{P}}\left[\left(F_{1}^{(\lambda)}\right)^{-p}\right]<+\infty .
$$


Proof. Choose conjugate exponents $(q, r)$ with $q \in(1, a T / p)$ and $r \in(a T /(a T-p), 2)$, and pick $\zeta \in(p q / a, T)$. Observe that

$$
\begin{aligned}
\mathbb{E}_{\mathbb{P}}\left[\left(F_{1}^{(\lambda)}\right)^{-p}\right] & \leq T^{p} \mu([\zeta, T])^{-p} \mathbb{E}_{\mathbb{P}}\left[\left(\frac{1}{\mu([\zeta, T])} \int_{\zeta}^{T} S_{t}^{(\lambda)} Y_{t}^{(\lambda)} \mu(d t)\right)^{-p}\right] \\
& \leq T^{p} \mu([\zeta, T])^{-p-1} \mathbb{E}_{\mathbb{P}}\left[\left(Y_{\zeta}^{(\lambda)}\right)^{-p q}\right]^{1 / q}\left(\int_{\zeta}^{T} \mathbb{E}_{\mathbb{P}}\left[\left(S_{t}^{(\lambda)}\right)^{-p r}\right] \mu(d t)\right)^{1 / r},
\end{aligned}
$$

where we have used the nonnegativity of the integrand, the nonnegativity and the nondecreasingness of the process $Y$, the Jensen inequality, and the Hölder inequality. Finally, the finiteness of the above last line follows from the moment formulas of Lemma 3.3.

We investigate the integrability of the random variable $F_{j}^{(\lambda)}, H_{j}^{(\lambda)}, I_{j}^{(\lambda)}$ and $J^{(\lambda)}$ of positive order.

Lemma 3.7. For $p \in\left(1, p_{0} / 2 \wedge(b-\varepsilon) /(2 \theta)\right)$, it holds that

$$
\sup _{\lambda \in B_{\varepsilon}} \mathbb{E}_{\mathbb{P}}\left[\sum_{j=0}^{3}\left(F_{j}^{(\lambda)}\right)^{p}+\sum_{j=0}^{1}\left(\left(H_{j}^{(\lambda)}\right)^{p}+\left(I_{j}^{(\lambda)}\right)^{p}\right)+\left(J^{(\lambda)}\right)^{p}\right]<+\infty .
$$

Proof. By choosing conjugate exponents $(q, r)$ and $(u, v)$ with $q \in(1, \sqrt{2})$ and $v \in(1, \sqrt{2})$, we have by the Jensen inequality,

$$
\begin{aligned}
& \mathbb{E}_{\mathbb{P}}\left[\sum_{j=0}^{3}\left(F_{j}^{(\lambda)}\right)^{p}+\sum_{j=0}^{1}\left(\left(H_{j}^{(\lambda)}\right)^{p}+\left(I_{j}^{(\lambda)}\right)^{p}\right)+\left(J^{(\lambda)}\right)^{p}\right] \\
& \leq c_{p} T^{-p} \mu([0, T])^{p} \mathbb{E}_{\mathbb{P}}\left[\left(\frac{1}{\mu[0, T]} \int_{0}^{T} S_{t}^{(\lambda)}\left(Y_{t}^{(\lambda)}\right)^{j} Z_{t}^{k} t^{l} \mu(d t)\right)^{p}\right] \\
& \leq c_{p} T^{l p-p} \mu([0, T])^{p-1} \mathbb{E}_{\mathbb{P}}\left[\left(Y_{T}^{(\lambda)}\right)^{j p q u}\right]^{\frac{1}{q u}} \mathbb{E}_{\mathbb{P}}\left[e^{p r Z_{T}}\right]^{1 / r} \int_{0}^{T} \mathbb{E}_{\mathbb{P}}\left[\left(S_{t}^{(\lambda)}\right)^{p q v}\right]^{\frac{1}{q v}} \mu(d t),
\end{aligned}
$$

which is well defined by Lemma 3.3 and Assumption 2.2 .

Proof of Theorem 3.1. We start with the smoothness assumption $\Phi \in C_{b}^{2}(\mathbb{R} ; \mathbb{R})$, which can be removed later by standard density arguments. (For details, we refer to Kawai and Kohatsu-Higa [13] and Kawai and Takeuchi [14].) Throughout the proof, we let $\lambda \in B_{\varepsilon}$.

(i) By Lemma2.1, it holds that

$$
\mathbb{E}_{\mathbb{P}}\left[\Phi\left(F_{0}^{(\lambda)}\right) \frac{F_{0}^{(\lambda)}}{S_{0} \theta F_{1}^{(\lambda)}}\right]=\mathbb{E}_{\mathbb{P}}\left[\frac{e^{\lambda Y_{T}}}{\mathbb{E}_{\mathbb{P}}\left[e^{\lambda Y_{T}}\right]} \Phi\left(F_{0}\right) \frac{F_{0}}{S_{0} \theta F_{1}}\right]
$$

Differentiating both sides of (3.1) at $\lambda=0$ yields the result, provided that the differentiation with respect to $\lambda$ and the expectation $\mathbb{E}_{\mathbb{P}}$ are commutative on the both hand sides, which we will prove 
in what follows. Concerning the left hand side of (3.1), by the Taylor theorem and Lemma 3.5 , it holds that

$$
\begin{aligned}
& \sup _{|\lambda| \leq \varepsilon}\left|\mathbb{E}_{\mathbb{P}}\left[\frac{1}{\lambda}\left(\Phi\left(F_{0}^{(\lambda)}\right) \frac{F_{0}^{(\lambda)}}{S_{0} \theta F_{1}^{(\lambda)}}-\Phi\left(F_{0}\right) \frac{F_{0}}{S_{0} \theta F_{1}}\right)-\left(\Phi^{\prime}\left(F_{0}\right) \frac{F_{0}}{S_{0} b}+\Phi\left(F_{0}\right) \frac{U^{(0)}}{S_{0} b}\right)\right]\right| \\
& \left.\leq \sup _{|\lambda| \leq \varepsilon} \int_{0}^{1} \mathbb{E}_{\mathbb{P}}\left[\mid \Phi^{\prime}\left(F_{0}^{(\delta \lambda)}\right) \frac{F_{0}^{(\delta \lambda)}}{S_{0}(b-\delta \lambda)}+\Phi\left(F_{0}^{(\delta \lambda)}\right) \frac{U^{(\delta \lambda)}}{S_{0}(b-\delta \lambda)}-\Phi^{\prime}\left(F_{0}\right) \frac{F_{0}}{S_{0} b}-\Phi\left(F_{0}\right) \frac{U^{(0)}}{S_{0} b}\right]\right] d \delta \\
& \leq \int_{0}^{1} \sup _{|\lambda| \leq \varepsilon} \mathbb{E}_{\mathbb{P}}\left[\left|\Phi^{\prime}\left(F_{0}^{(\delta \lambda)}\right) \frac{F_{0}^{(\delta \lambda)}}{S_{0}(b-\delta \lambda)}-\Phi^{\prime}\left(F_{0}\right) \frac{F_{0}}{S_{0} b}\right|\right] d \delta \\
& \quad+\int_{0}^{1} \sup _{|\lambda| \leq \varepsilon} \mathbb{E}_{\mathbb{P}}\left[\left|\Phi\left(F_{0}^{(\delta \lambda)}\right) \frac{U^{(\delta \lambda)}}{S_{0}(b-\delta \lambda)}-\Phi\left(F_{0}\right) \frac{U^{(0)}}{S_{0} b}\right|\right] d \delta,
\end{aligned}
$$

where

$$
U^{(\lambda)}:=1-\frac{F_{0}^{(\lambda)} F_{2}^{(\lambda)}}{\left(F_{1}^{(\lambda)}\right)^{2}}-\frac{F_{0}^{(\lambda)}}{\theta F_{1}^{(\lambda)}} .
$$

The first integral on the right hand side tends to zero as $\varepsilon \downarrow 0$ by the Cauchy-Schwarz inequality with the boundedness of $\Phi^{\prime}$ and Lemma 3.4. For the second integral, again by the CauchySchwarz inequality with $F_{2}^{(\lambda)} \leq Y_{T}^{(\lambda)} F_{1}^{(\lambda)}, \mathbb{P}$-a.s., it remains to show that for $\lambda \in B_{\varepsilon}$ and for $k=0,1, \mathbb{E}_{\mathbb{P}}\left[\left(F_{0}^{(\lambda)}\left(Y_{T}^{(\lambda)}\right)^{k} / F_{1}^{(\lambda)}\right)^{2}\right]<+\infty$. By choosing conjugate exponents $(p, q, r)$ with $p \in$ $\left(1,\left(p_{0} \wedge a T\right) / 4\right)$ and $q \in\left(1, p_{0} / 4 \wedge(b-\varepsilon) /(4 \theta)\right)$, we have

$$
\mathbb{E}_{\mathbb{P}}\left[\left(\frac{F_{0}^{(\lambda)}\left(Y_{T}^{(\lambda)}\right)^{k}}{F_{1}^{(\lambda)}}\right)^{2}\right] \leq \mathbb{E}_{\mathbb{P}}\left[\left(F_{1}^{(\lambda)}\right)^{-2 p}\right]^{1 / p} \mathbb{E}_{\mathbb{P}}\left[\left(F_{0}^{(\lambda)}\right)^{2 q}\right]^{1 / q} \mathbb{E}_{\mathbb{P}}\left[\left(Y_{T}^{(\lambda)}\right)^{2 k r}\right]^{1 / r}
$$

which is uniformly bounded over $|\lambda| \leq \varepsilon$ by Lemma 3.3, Lemma 3.6 and Lemma 3.7. The interchange of the derivative with respect to $\lambda$ and the expectation $\mathbb{E}_{\mathbb{P}}$ on the right hand side of (3.1) can be shown by the Cauchy-Schwarz inequality with $\mathbb{E}_{\mathbb{P}}\left[\left|\Phi\left(F_{0}\right)\right|^{2}\right]<+\infty$ and with

$$
\frac{e^{\lambda Y_{T}-\varphi_{T}(\lambda)}-1}{\lambda} \rightarrow Y_{T}-\frac{a T}{b}
$$

in $\mathbb{L}^{2}(\Omega)$, as $\lambda \downarrow 0$.

(ii) Define $\Psi(x):=\Phi^{\prime}(x) x$. Observe that

$$
\begin{aligned}
\frac{\partial^{2}}{\partial S_{0}^{2}} \mathbb{E}_{\mathbb{P}}\left[\Phi\left(F_{0}\right)\right] & =\frac{\partial}{\partial S_{0}} \mathbb{E}_{\mathbb{P}}\left[\Phi^{\prime}\left(F_{0}\right) \frac{F_{0}}{S_{0}}\right]=\mathbb{E}_{\mathbb{P}}\left[\Phi^{\prime \prime}\left(F_{0}\right)\left(\frac{F_{0}}{S_{0}}\right)^{2}\right] \\
& =\frac{1}{S_{0}^{2}} \mathbb{E}_{\mathbb{P}}\left[\left(\Psi^{\prime}\left(F_{0}\right)-\Phi^{\prime}\left(F_{0}\right)\right) F_{0}\right]=\frac{1}{S_{0}^{2}} \mathbb{E}_{\mathbb{P}}\left[\left(\Psi\left(F_{0}\right)-\Phi\left(F_{0}\right)\right) K\right]
\end{aligned}
$$


where the first and the fourth equalities holds by the result (i), the second by Lemma 3.5, and the third by $\Phi^{\prime \prime}(x) x^{2}=\Psi^{\prime}(x) x-\Phi^{\prime}(x) x$. The desired result can be derived by taking derivative at $\lambda=0$ of the identity

$$
\mathbb{E}_{\mathbb{P}}\left[\Phi\left(F_{0}^{(\lambda)}\right) \frac{b F_{0}^{(\lambda)}}{\theta F_{1}^{(\lambda)}} K^{(\lambda)}\right]=\mathbb{E}_{\mathbb{P}}\left[\frac{e^{\lambda Y_{T}}}{\mathbb{E}_{\mathbb{P}}\left[e^{\left.\lambda Y_{T}\right]}\right.} \Phi\left(F_{0}\right) \frac{b F_{0}}{\theta F_{1}} K\right],
$$

where the interchange of the differentiation with respect to $\lambda$ and the expectation $\mathbb{E}_{\mathbb{P}}$ and the existence of the formulas can be justified in a similar manner to the proof of (i).

(iii)-(iv) The desired formulas can be derived by taking derivative at $\lambda=0$ respectively of

$$
\begin{gathered}
\mathbb{E}_{\mathbb{P}}\left[\Phi\left(F_{0}^{(\lambda)}\right)\left(1+\partial_{1} c(\theta, \kappa) \frac{I_{0}^{(\lambda)}}{F_{1}^{(\lambda)}}\right)\right]=\mathbb{E}_{\mathbb{P}}\left[\frac{e^{\lambda Y_{T}}}{\mathbb{E}_{\mathbb{P}}\left[e^{\left.\lambda Y_{T}\right]} \Phi\left(F_{0}\right)\left(1+\partial_{1} c(\theta, \kappa) \frac{I_{0}}{F_{1}}\right)\right],}\right. \\
\mathbb{E}_{\mathbb{P}}\left[\Phi\left(F_{0}^{(\lambda)}\right) \frac{H_{0}^{(\lambda)}+\partial_{2} c(\theta, \kappa) J^{(\lambda)}}{F_{1}^{(\lambda)}}\right]=\mathbb{E}_{\mathbb{P}}\left[\frac{e^{\lambda Y_{T}}}{\mathbb{E}_{\mathbb{P}}\left[e^{\lambda Y_{T}}\right]} \Phi\left(F_{0}\right) \frac{H_{0}+\partial_{2} c(\theta, \kappa) J}{F_{1}}\right] .
\end{gathered}
$$

We omit justifying the interchange of the derivative with respect to $\lambda$ and the expectation $\mathbb{E}_{\mathbb{P}}$ and the proof of the existence of the formulas.

We next present Greeks formulas for geometrically averaged asset price dynamics. Fix $N \in \mathbb{N}$ and $0<t_{1} \leq t_{2} \leq \cdots \leq t_{N} \leq T$. Define

$$
G^{(\lambda)}:=\sqrt[N]{S_{t_{1}}^{(\lambda)} S_{t_{2}}^{(\lambda)} \cdots S_{t_{N}}^{(\lambda)}}=S_{0} \exp \left[\theta \widetilde{Y}^{(\lambda)}+\kappa \widetilde{Z}+c(\theta, \kappa) \widetilde{t}\right]
$$

where $\widetilde{Y}^{(\lambda)}:=\sum_{k=1}^{N} Y_{t_{k}}^{(\lambda)} / N, \widetilde{Z}:=\sum_{k=1}^{N} Z_{t_{k}} / N, \widetilde{t}:=\sum_{k=1}^{N} t_{k} / N$. Without essential loss of generality, we have ruled out the case $t_{1}=0$ a priori. Notice that the above geometric averaging framework reduces to the European setting by putting $N=1$ and $t_{N}=T$. As before, we suppress the superscript $(0)$, that is, $\widetilde{Y}:=\widetilde{Y}^{(0)}$ and $G:=G^{(0)}$. Then, Greeks formulas are as follows.

Theorem 3.8. Let $\Phi: \mathbb{R}_{+} \rightarrow \mathbb{R}$ be a measurable function such that $\mathbb{E}_{\mathbb{P}}\left[|\Phi(G)|^{2}\right]$ is locally uniformly bounded in $S_{0}, \theta$ and $\kappa$. If $a t_{N}>2$, it holds that

$$
\begin{aligned}
\frac{\partial}{\partial S_{0}} \mathbb{E}_{\mathbb{P}}[\Phi(G)] & =\frac{1}{S_{0}} \mathbb{E}_{\mathbb{P}}\left[\Phi(G) \frac{b Y_{T}-a T+1}{\theta \widetilde{Y}}\right], \\
\frac{\partial}{\partial \theta} \mathbb{E}_{\mathbb{P}}[\Phi(G)] & =\mathbb{E}_{\mathbb{P}}\left[\Phi(G) \frac{b Y_{T}-a T}{\theta}\right]+S_{0} \partial_{1} c(\theta, \kappa) \widetilde{t} \frac{\partial}{\partial S_{0}} \mathbb{E}_{\mathbb{P}}[\Phi(G)], \\
\frac{\partial}{\partial \kappa} \mathbb{E}_{\mathbb{P}}[\Phi(G)] & =\mathbb{E}_{\mathbb{P}}\left[\Phi(G) \frac{\left(b Y_{T}-a T+1\right) \widetilde{Z}}{\theta \widetilde{Y}}\right]+S_{0} \partial_{2} c(\theta, \kappa) \widetilde{t} \frac{\partial}{\partial S_{0}} \mathbb{E}_{\mathbb{P}}[\Phi(G)] .
\end{aligned}
$$


If $a t_{N}>4$, then it holds that

$$
\frac{\partial^{2}}{\partial S_{0}^{2}} \mathbb{E}_{\mathbb{P}}[\Phi(G)]=\frac{1}{S_{0}^{2}} \mathbb{E}_{\mathbb{P}}\left[\Phi(G) \frac{\left(b Y_{T}-a T+1\right)^{2}+a T-1+2 \theta^{2}\left(1-\widetilde{Y}^{2}\right)}{\theta^{2} \widetilde{Y}^{2}}\right]-\frac{1}{S_{0}} \frac{\partial}{\partial S_{0}} \mathbb{E}_{\mathbb{P}}[\Phi(G)] .
$$

Proof. To avoid overloading this proof with nonessential details, we only give the proof of the Delta formula at a sketchy level. With the additional regularity assumption $\Phi \in C_{b}^{2}(\mathbb{R} ; \mathbb{R})$, we get the formula by differentiating the identity

$$
\mathbb{E}_{\mathbb{P}}\left[\frac{\Phi\left(G^{(\lambda)}\right)}{\widetilde{Y}(\lambda)}\right]=\mathbb{E}_{\mathbb{P}}\left[\frac{e^{\lambda Y_{T}}}{\mathbb{E}_{\mathbb{P}}\left[e^{\lambda Y_{T}}\right]} \frac{\Phi(G)}{\widetilde{Y}}\right]
$$

at $\lambda=0$. The interchange of the derivative with respect to $\lambda$ and the expectation $\mathbb{E}_{\mathbb{P}}$ and the removal of the regularity of $\Phi$ can be justified as before, with the help of

$$
\mathbb{E}_{\mathbb{P}}\left[Y_{T}^{2} / \widetilde{Y}^{2}\right] \leq \mathbb{E}_{\mathbb{P}}\left[Y_{T}^{2} / Y_{t_{N}}^{2}\right]=\mathbb{E}_{\mathbb{P}}\left[\left(Y_{T}-Y_{t_{N}}\right)^{2}\right] \mathbb{E}_{\mathbb{P}}\left[Y_{t_{N}}^{-2}\right]+2 \mathbb{E}_{\mathbb{P}}\left[Y_{T}-Y_{t_{N}}\right] \mathbb{E}_{\mathbb{P}}\left[Y_{t_{N}}^{-1}\right]+1<+\infty,
$$

where we have used the independence of the increments.

Let us close our study with numerical examples to illustrate the performance of our Greeks formulas in Monte Carlo estimations. We consider a digital payoff $\Phi\left(F_{0}\right)=\mathbb{1}\left(F_{0}>C\right)$, for some $C>0$, with the weighting measure $\mu(d t)=T N^{-1} \sum_{k=1}^{N} \delta_{k \frac{T}{N}}(d t)$, so that $\Phi\left(F_{0}\right)$ is an equidistant discrete Asian option. We divide $[0, T]$ into $N=20$ intervals. Let us emphasize that as discussed in Remark 3.2, our approach is rigorously valid for this discrete Asian options, not as a numerical approximation of continuously averaged Asians. Fix $S_{0}=100, C=110, T=1$, $a=b=6$ and $\theta=1$. In our experiments, the process $Z$ is an inverse Gaussian (Lévy) process, which is introduced in Remark 2.3. We set $\kappa=-1$ and $v=30$. Concerning the generation of a inverse Gaussian process, we follow an algorithm proposed in Rydberg [16]. By setting $c(\theta, \kappa)=a \ln (1-\theta / b)+\left(\sqrt{-2 \kappa+v^{2}}-v\right)$, we can induce the model risk-neutral. For simplicity, we omit the risk-free discounting. Figure 1 presents numerical results of the sensitivities $\partial / \partial S_{0}$, $\partial^{2} / \partial S_{0}^{2}, \partial / \partial \theta$ and $\partial / \partial \kappa$ of the expectation $\mathbb{E}_{\mathbb{P}}\left[\Phi\left(F_{0}\right)\right]$. For clear comparisons, the convergence of the finite difference method is also provided. The figures and the variance ratios evidently indicate a faster convergence of our Greeks formulas. Note also that in general, the finite difference approximation yields some bias.

\section{Acknowledgements}

The authors are grateful to Co-Editor-in-Chief Prof.Hira Koul for his careful reading and valuable suggestions. This work was supported in part by JSPS Grant-in-Aid for Scientific Research 

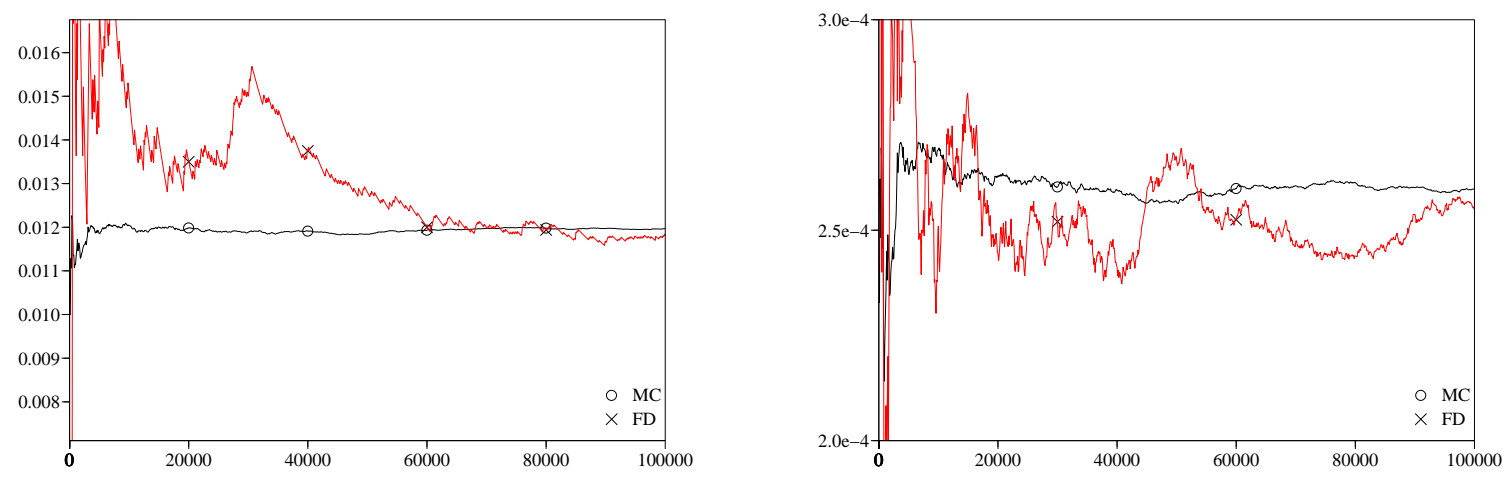

$\frac{\partial}{\partial S_{0}} \mathbb{E}_{\mathbb{P}}\left[\Phi\left(F_{0}\right)\right]$, vratio $=144$ and $\varepsilon=1 \mathrm{e}-3$

$\frac{\partial^{2}}{\partial S_{n}^{2}} \mathbb{E}_{\mathbb{P}}\left[\Phi\left(F_{0}\right)\right]$, vratio $=33$ and $\varepsilon=1 \mathrm{e}-1$
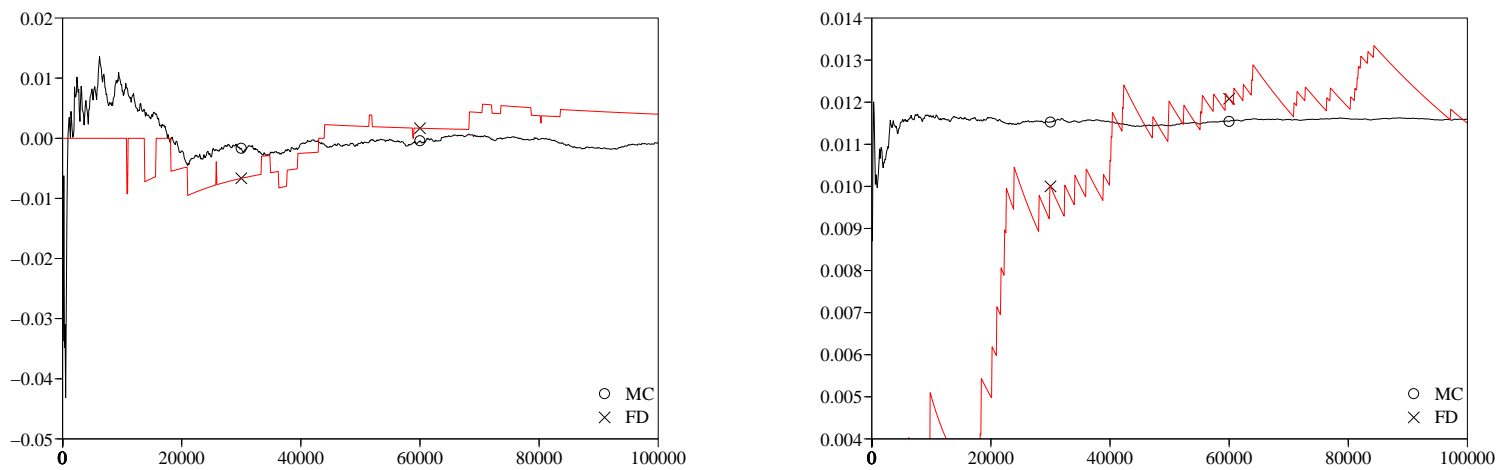

$\frac{\partial}{\partial \theta} \mathbb{E}_{\mathbb{P}}\left[\Phi\left(F_{0}\right)\right]$, vratio $=9$ and $\varepsilon=5 \mathrm{e}-3$

$\frac{\partial}{\partial \kappa} \mathbb{E}_{\mathbb{P}}\left[\Phi\left(F_{0}\right)\right]$, vratio $=366$ and $\varepsilon=2 \mathrm{e}-2$.

Figure 1: "vratio" indicates the variance ratio $\frac{\operatorname{Var}(\text { Finite Difference })}{\operatorname{Var}(\text { Malliavin Calculus })}$. The quantity $\varepsilon$ is the increment in the finite difference estimation $\frac{X((1+\varepsilon) \theta)-X((1-\varepsilon) \theta)}{2 \varepsilon \theta}$ or $\frac{X((1+\varepsilon) \theta)-2 X(\theta)+X((1-\varepsilon) \theta)}{(\varepsilon \theta)^{2}}$.

20740059, and was largely carried out while RK was based at Center for the Study of Finance and Insurance, Osaka University, Japan.

\section{References}

[1] Bally, V., Bavouzet, M., Messaoud, M. (2007) Integration by parts formula for locally smooth laws and applications to sensitivity computations, Ann. Appl. Probab., 17, 33-66.

[2] Barndorff-Nielsen, O.E. (1998) Processes of normal inverse Gaussian type. Finance Stoch., 2, 41-68. 
[3] Bichteler, K., Gravereaux, J. B., Jacod, J. (1987) Malliavin calculus for processes with jumps, Gordon and Breach Science Publishers, New York.

[4] Bismut, J.M. (1983) Calcul des variations stochastique et processus de sauts. Z. Wahrsch. Verw. Gebiete, 63, 147-235.

[5] Carlen, E., Pardoux, E. (1990) Differential calculus and integration by parts on Poisson space, In: Albeverio, S., Blanchard, Ph., Testard, D. (Eds.), Stochastics, algebra and analysis in classical and quantum dynamics, Kluwer Acad. Publ., pp. 63-73.

[6] Carr, P., Geman, H., Madan, D., Yor, M. (2003) Stochastic Volatility for Lévy processes. Math. Finance, 13, 345-382.

[7] Carr, P., Madan, D. (1999) Option pricing and the fast Fourier transform. J. Comput. Finance, 2, 61-73.

[8] Cass, T. R., Friz, P. K. (2007) The Bismut-Elworthy-Li formula for jump-diffusions and applications to Monte Carlo methods in finance, available at arXiv:math/0604311v3.

[9] Davis, M.H.A., Johansson, M.P. (2006) Malliavin Monte Carlo Greeks for jump diffusions. Stochastic Process. Appl., 116, 101-129.

[10] El-Khatib, Y., Privault, N. (2004) Computations of Greeks in a market with jumps via the Malliavin calculus. Finance Stoch., 8, 161-179.

[11] Fournié, E., Lasry, J., Lebuchoux, J., Lions, P., Touzi, N. (1999) Applications of Malliavin calculus to Monte Carlo methods in finance. Finance Stoch., 3, 391-412.

[12] Fu, M.C. (2007) Variance-Gamma and Monte Carlo, In: Fu, M. C., Jarrow, R. A., Yen, J.-Y. J., Elliott, R. J. (Eds.), Advances in Mathematical Finance, Birkhäuser, pp. 21-35.

[13] Kawai, R., Kohatsu-Higa, A. (2009) Computation of Greeks and multidimensional density estimation for asset price models with time-changed Brownian motion, to appear in Appl. Math. Finance.

[14] Kawai, R., Takeuchi, A. (2009) Greeks formulae for an asset price model with gamma processes, under revision.

[15] Madan, D., Carr, P., Chang, E. (1998) The variance gamma process and option pricing. European Finance Review, 2, 79-105. 
[16] Rydberg, T.H. (1997) The normal inverse Gaussian Lévy process: simulation and approximation. Comm. Statist. Stochastic Models, 13, 887-910.

[17] Takeuchi, A. (2009) The Bismut-Elworthy-Li type formulae for stochastic differential equations with jumps, under revision. 